\title{
A LA NATURALEZA SE LA RESPETA
}

\author{
Cristóbal Bellolio \\ Universidad Adolfo lbáñez
}

\begin{abstract}
A sediado por las fuerzas policiales y sus adversarios en el negocio de la droga, Pablo Escobar Gaviria resiste en la selva. Por más que lo intentan, no pueden doblegarlo. Es una fuerza indomable en su territorio. Desde el teléfono, les advierte a sus enemigos: "A Pablo Escobar se le respeta". Se me vino a la cabeza la escena - tomada de la serie Narcos - a propósito del nuevo libro de Álvaro Fischer que lleva por título De naturaleza liberal (Santiago: Catalonia, 2017). Asediada por el ímpetu transformador de la cultura y la vocación utópica de ciertas ideologías políticas, la naturaleza resiste. No es cosa de llegar y cambiar la matriz de la especie humana. Algunos elementos son de fábrica. A veces yacen dormidos, pero despiertan cuando las pistas del medio ambiente lo sugieren. Con la naturaleza, en definitiva, no se juega. Y cada vez que se intenta, el resultado es tan infructuoso y frustrante como la cacería de Escobar. Esta analogía, sin embargo, se queda corta: el "patrón" colombiano fue finalmente abatido y su imperio, desmembrado. La natura, en cambio, no da señales de fatiga. Salvo que volquemos nuestros esfuerzos a modificar la genética humana a gran escala, lo más probable es que nuestra arquitectura original, labrada por millones de años de evolución, no sufra más que abollones menores tras el embate de la cultura.
\end{abstract}

Cristóbal Bellolio. Abogado y licenciado en ciencia política de la Pontificia Universidad Católica de Chile. Doctor en filosofía política por la University College London, Reino Unido. Profesor asistente de la Escuela de Gobierno de la Universidad Adolfo Ibáñez. Email: cristobal.bellolio@uai.cl. 
Éste es el argumento central del libro de Fischer. Es un argumento provocador por varias razones. Primero, porque el autor introduce la variable del saber científico para constreñir el ámbito de las intervenciones posibles en el campo político y económico, lo que aparentemente viola la llamada Ley de Hume. Segundo, porque recurre al mismo paradigma evolucionario para explicar la emergencia del orden tanto en la naturaleza como en la vida económica, como si cada uno sirviera de evidencia para probar al otro. Tercero, porque limita considerablemente el espacio del debate normativo y las consideraciones constructivistas sobre la justicia, sobre las cuales descansa buena parte del ideario liberal contemporáneo. Y cuarto, porque reduce al socialismo a una maladaptación. Paso a revisar estos temas a continuación.

\section{LA LEY DE HIERRO DE LA SOCIOBIOLOGÍA}

Álvaro Fischer es un conocedor del estado del arte en psicología evolucionaria, extensión disciplinaria de lo que alguna vez se conoció como sociobiología. En su libro desfilan los trabajos de Robert Axelrod, Leda Cosmides, Jonathan Haidt, William Hamilton, Steven Pinker, Robert Trivers y E.O. Wilson, entre otros. La conclusión de este proyecto intelectual apunta a que no somos una página en blanco susceptible de ser rellenada a voluntad. Por el contrario, nuestra sensibilidad moral y disposición conductual es parte de la herencia que hemos recibido como especie. Dicho de otro modo, nuestra mirada ética es innata antes que aprendida. Así, encriptada en nuestra arquitectura neuronal, sesga las posibilidades de interacción social. Esto no quiere decir que la naturaleza elimine la posibilidad de innovación cultural, pero sí la restringe en forma relevante. La evolución puramente cultural no tendría el poder de alterar significativamente nuestras herramientas mentales originarias. De ahí, infiere Fischer, que la cultura se subordina a la naturaleza humana y no al revés.

¿Cuál es la implicancia de lo anterior? Que hay que cuidarse de promover reglas y normas que contradigan los impulsores conductuales básicos de la naturaleza humana. Aunque es posible intuir que estas reglas y normas provocarán tensiones que a su vez generarán grados de sufrimiento, el argumento recurrente de Fischer es que las personas 
encontrarán su manera de esquivarlas. $\mathrm{O}$, como diría el escéptico personaje de Jeff Goldblum en la película hollywoodense Jurassic Park, la vida siempre se abre camino. La posición de la psicología evolutiva, en este sentido, colisiona al menos parcialmente con las aspiraciones normativas de las ideologías que buscan modelar el comportamiento de las personas a través del diseño deliberado de instituciones sociales, políticas y económicas. Choca, sobre todo, con la pretensión de parir un hombre nuevo sobre la faz de la Tierra.

La pregunta metodológica es si acaso es legítimo introducir un argumento de índole científica en el debate normativo. La sospecha es que Fischer viola la Ley de Hume, que separa estrictamente la discusión sobre aquello que es y aquello que debe ser. La ciencia participa en la primera. La política, en la segunda. Sin embargo, el autor propone que las conclusiones obtenidas en la primera discusión afectan la segunda. Esto no es nuevo. La empresa intelectual conocida como darwinismo social — asociada a la figura de Herbert Spencer y luego al proyecto eugenésico de Francis Galton y los nazis- fue tristemente célebre justamente por violar la Ley de Hume y perpetrar la llamada falacia naturalista. Los promotores del darwinismo social pensaron que la dinámica evolucionaria escondía un patrón que, una vez descubierto, debía ser imitado por las instituciones humanas. De ese modo, extendieron la noción de la supervivencia del más fuerte a nuestras relaciones políticas, económicas y sociales.

Sería injusto, sin embargo, confundir la psicología evolucionaria como disciplina con el mal afamado darwinismo social. Aunque hay pensadores que sostienen que hay algo intrínsecamente perverso en recurrir a argumentos científicos sobre nuestra naturaleza para terciar en debates filosóficos o políticos (Midgley 2002), lo cierto es que es difícil pensar en ambas discusiones como completamente separadas e independientes. La Ley de Hume se sostiene en el sentido estricto de que no se puede colegir automáticamente una prescripción de una descripción. Pero lo que hacen Fischer y sus colegas en el campo de la sociobiología es sutilmente distinto: llaman la atención respecto de la viabilidad y deseabilidad de ciertas prescripciones que ignoran el conocimiento actual respecto de nuestra constitución fundamental como especie. Esto será normalmente interpretado como una limitante ante los ojos del pensamiento utópico, que no se contenta con la realidad y desea fervien- 
temente transformarla (de ahí la undécima tesis sobre Feuerbach que desfila orgullosa en el acervo marxista). Pero, en sí misma, no constituye una falacia naturalista, pues la psicología evolutiva no hace proselitismo en torno a los mecanismos de la naturaleza como si éstos fuesen moralmente buenos y por ende dignos de imitación. En cambio, lo que reclama Fischer es que la política entienda y respete las características duras de la naturaleza humana. En cierto modo, esto sí constituye una apostasía del mandato humeano de separar hechos y valores. Pero, en tanto Fischer declara su simpatía hacia el proyecto de E.O. Wilson (1998) de unificar el conocimiento de las distintas ciencias y humanidades, es una apostasía que debiese asumirse en forma impenitente.

\section{LA CONEXIÓN SMITH-DARWIN}

En defensa de la libertad - especial, pero no exclusivamente económica-, el autor produce el siguiente argumento: con el objetivo de incrementar nuestras opciones reproductivas — pulsión innata no sólo de nuestra especie, sino que también de muchos otros animales-, los seres humanos estamos en continua búsqueda de mejorar nuestro estatus frente a nuestros pares. Esta competencia por estatus, inevitablemente, nos lleva a querer diferenciarnos más que igualarnos. La institución social idónea para dicha pulsión básica será entonces la que reconozca ese afán diferenciador. En el ámbito económico, por ejemplo, será una economía de mercado. En oposición a la crítica de la izquierda - que acusa a dicho modelo de haber sido impuesto a la fuerza y en cierta forma "naturalizado" desde el discurso político-, Fischer sostiene que las leyes de dicho orden son efectivamente naturales pues se conectan de mejor manera con nuestras tendencias innatas.

La libertad altera todos los patrones, escribió Robert Nozick (1974). Que nadie la detenga, agregaría Fischer. Su libro no pretende ocultar las propiedades subversivas de la libertad. Por el contrario, las celebra. Cuando hay libertad, se produce desigualdad. Eso no es necesariamente problemático para el liberalismo de cuño darwinista del autor. La desigualdad es testimonio de nuestro motor diferenciador, que a su vez obedece al imperativo biológico de la búsqueda de estatus, como dijimos, para mejorar nuestras opciones reproductivas. Las maneras de 
obtener dicho estatus son variadas. En algunos casos y épocas será la fuerza, en otros la riqueza, en otros el poder, en otros la casta, en otros la estética, etcétera. Lo relevante es que las personas sean libres de escoger sus estrategias. Si se les prohíbe la diferenciación (castigando ciertas estrategias) en nombre de la igualdad, se contradice la naturaleza. Por eso es necesario, piensa Fischer, que el valor de la libertad sea privilegiado en la estructura normativa de la sociedad. La libertad altera los patrones porque, justamente, de eso se trata. En palabras de Fischer, "si uno de los impulsores básicos de nuestra conducta social es nuestro afán por diferenciarnos más que por igualarnos, ¿por qué habríamos de preferir la igualdad?" $(2017,221){ }^{1}$

La libertad económica y su efecto subversivo, entonces, tendrían un fundamento natural. Es un argumento que, como reconoce el autor, estaba ya presente en Adam Smith, quien creía que el intercambio económico era una función natural de la especie humana. El orden que se produce, en consecuencia, no es producto de un plan central deliberado. Es, en la terminología hayekiana, espontáneo. Nadie lo diseñó ex ante. Emerge del caos de las relaciones humanas y encuentra su equilibrio sin necesidad de intervención de ningún poder superior. Lo mismo, advierte Fischer, ocurre en el orden de la naturaleza. El cosmos y la rica biodiversidad del planeta que habitamos no fueron diseñados por ninguna mente superinteligente. La mano invisible de Smith encontraría su símil en la selección natural de Charles Darwin: ambos serían órdenes emergentes que no necesitaron un planificador central pues alcanzan su equilibrio, económico y ecológico respectivamente, en un infinito juego de ensayo y error entre los diversos agentes que participan en ellos. Sin ir más lejos, F.A. Hayek fundó su liberalismo en esta reflexión epistemológica. En específico, Hayek (1960) subrayó que no fue Darwin sino los filósofos de la ilustración escocesa quienes revolucionaron la manera de pensar las instituciones sociales, ya no de-arriba-hacia-abajo, sino deabajo-hacia-arriba. La acción humana, sostenía en este contexto Adam Ferguson, no es lo mismo que el diseño humano. Darwin, insistió Hayek, se apoyó intelectualmente en Smith y compañía para desarrollar su teoría. Fischer, en tanto, recurre a Darwin para mostrar que los órdenes sin planificación central no son sólo posibles, sino que naturales.

\footnotetext{
${ }^{1}$ En adelante, el libro de Fischer se citará tan sólo con la página en paréntesis.
} 
Esta conexión entre Smith y Darwin ha generado históricamente cierta incomodidad en ambos extremos del espectro político. Smith ha sido apropiado por la derecha en función de su predilección por los mercados libres y los gobiernos limitados, pero esos mismos círculos rechazan la teoría de Darwin por sus complejas implicancias teológicas. En Estados Unidos, por ejemplo, no es extraño encontrar libertarios económicos que simultáneamente son creacionistas. En contrapartida, la teoría de la evolución por selección natural es comúnmente abrazada por la izquierda — sin ir más lejos, fue originalmente celebrada por Marx y Engels, pues le daba un golpe de gracia al discurso teleológico de la religión-, que a su vez promueve políticas económicas que no tienen nada que ver con la mecánica descentralizada que caracteriza al proceso darwiniano. Aunque esta alineación — Smith para la derecha, Darwin para la izquierda - puede parecer caduca, la crítica de inconsistencia filosófica que esta alineación sugiere se ha renovado con fuerza en los últimos años (Arnhart 2005; Shermer 2006; Ridley 2012). Sin embargo, ante la posible crítica de inconsistencia - que Álvaro Fischer no hace expresamente, pero que se desprende de su argumento-, tanto creacionistas como marxistas podrían argüir que la similitud entre ambos órdenes es trivial, pues discurren en planos distintos: Dios podría haber creado cada especie en su forma actual y eso no sería obstáculo filosófico para endosar una economía de mercado. Del mismo modo, la biodiversidad podría haber surgido de acuerdo con la tesis darwiniana y eso no sería objeción filosófica en contra de la organización de un sistema económico bajo control estatal.

Lo anterior no implica que la conexión Smith-Darwin sea espuria. Sigue siendo una conexión potente, aunque en un sentido ligeramente diverso: si bien sugiere que tanto las instituciones sociales como las realidades ecológicas son empresas acumulativas, que se construyen sobre información dispersa y desagregada, su contribución específicamente política apunta a la existencia de ciertas tendencias innatas y disposiciones conductuales naturales que no serán fácilmente derogadas por intervención cultural. Paradójicamente, fue justamente Hume (1739) quien señaló que lo máximo a lo que los políticos podían aspirar era a proyectar los sentimientos naturales del ser humano, pero tomando siempre en consideración que la naturaleza es la que provee los materiales originales y entrega las nociones morales básicas. En este 
registro, la ilustración escocesa y la psicología evolucionaria parecen estar de acuerdo: a la naturaleza se la respeta. Aparentemente, Hume no estaría enteramente comprometido con su propia ley, pues, al igual que Fischer, parece condicionar los debates normativos a las posibilidades y limitaciones de la naturaleza humana. Para salvar la aparente contradicción, ambos podrían argumentar que no es lo mismo sostener que (a) lo normativo se deriva de lo descriptivo - que es lo que prohíbe la Ley de Hume - que sostener que (b) la naturaleza condiciona las posibilidades de éxito de la acción política (que no viola necesariamente la Ley de Hume).

\section{CONTRA EL LIBERALISMO RACIONALISTA}

La preferencia de Fischer por Hume no termina aquí. Mientras Kant señalaba que la libertad consiste en sobreponerse a las pasiones e inclinaciones naturales para elevarse a la categoría de criatura racional, Hume pensaba que la razón actuaba como sirviente de nuestras emociones básicas. En otras palabras, mientras Kant articulaba la noción de libertad moral como libertad de la naturaleza, Hume sostenía que cualquier libertad relevante se despliega dentro de la naturaleza. Como buen darwinista, Fischer endosa la segunda tesis. Es más, siguiendo a Haidt (2012), destaca que los argumentos que desplegamos en nuestras conversaciones cotidianas y debates políticos no son realmente productos de una reflexión racional, sino que más bien la razón se encarga de justificar una emoción o sentimiento que es anterior al proceso racional y que determina a fin de cuentas nuestra posición.

Ése sería el principal obstáculo que enfrentarían las teorías racionalistas sobre la justicia, para las cuales es posible arribar a una definición respecto a lo justo sobre la confianza en que los individuos están tomando decisiones racionales. Es, sin ir más lejos, la esperanza del proyecto rawlsiano — de inspiración kantiana - que ha dominado la teoría política liberal en los últimos cuarenta años. Sería más prometedor, arguye Fischer, implementar reglas que reconozcan nuestras pulsiones innatas. La naturaleza humana es más porfiada que los imperativos categóricos. Su propuesta, entonces, es ponerse del lado de las fuerzas de la naturaleza antes que luchar contra ellas cuando se trata de diseñar políticas y normas: "Más que estar en permanente combate 
o negación", concluye Fischer, "nos conviene utilizar los rasgos característicos de la naturaleza humana, porque ellos están encriptados en nuestro genoma y no se modifican por persuasión ni por decreto" (280). El autor recurre en un par de ocasiones a la metáfora del judo: si pensamos que la naturaleza es nuestra adversaria, tenemos que ser capaces de usar su fuerza a nuestro favor.

Esto es claro en el caso de nuestro espíritu competitivo y el afán diferenciador: hay que darle cauce antes que restringirlo, piensa Fischer. Por eso conviene que la regulación de la actividad económica sea limitada. También parece claro en aquellos casos en los cuales hemos penalizado ciertas conductas que nuestra inteligencia evolucionaria ha identificado como dañinas, como el incesto. Pero hay otros casos más complejos. El autor reconoce que nuestro cableado neuronal también incluye una tendencia a formar grupos y a excluir a aquellos que no son parte de la tribu. Sería entonces natural mantener relaciones adversariales con los hinchas del equipo rival, los militantes del partido político opuesto, los fieles de una religión distinta. Sin embargo, pocas cosas generan más tribalismo que la raza. El racismo, desde este punto de vista, también sería una expresión natural. ¿Significa este reconocimiento que hay que legitimarlo en el espacio político? Fischer tiene una coartada a mano: la división racial es una construcción cultural, pues nuestra especie no estaba aún dividida en razas cuando nuestro entramado neuronal quedó fijado. Es una salida plausible, pero da cuenta de un problema latente en la teoría: ¿qué hacer cuando no haya coartada disponible? ¿Admitir, sin más, que la naturaleza manda y que el rol de la política es buscar la forma de adaptarse a ella para darle cauce?

Piénsese en el caso del nepotismo. Fischer indica correctamente que estamos predispuestos por naturaleza a beneficiar a nuestros seres queridos, en especial a aquéllos con quienes compartimos identidad genética. Siempre queremos lo mejor para nuestros hijos. Esto explicaría, según el autor, por qué las políticas educacionales tendientes a uniformar — por ejemplo, la que elimina la posibilidad del copago en los colegios particulares subvencionados- están destinadas a fracasar. Es antinatural, por decirlo de alguna forma, exigirles a los padres que no traten de aventajar a sus hijos en la carrera de la vida (lo que implica, en la práctica, entregarles mejores opciones que a los hijos del vecino). Pero la misma tendencia puede reconocerse en otras esferas de la vida. 
Por ejemplo, sería natural que los gobernantes ubicaran a sus parientes en posiciones de poder e influencia. Sin embargo, esto nos hace ruido. Nepotismo no es precisamente un término de elogio. Por eso elaboramos reglas para evitar que se produzcan dichas situaciones. ¿Deberíamos desprendernos de dichas reglas por el hecho de contravenir una inclinación tan arraigada en la conducta del ser humano?

En cualquier caso, las tensiones entre el proyecto liberal-igualitario de cuño rawlsiano y el tipo de liberalismo evolutivo que defiende Fischer no deben ser exageradas. Fischer admite que las personas manifiestan la disposición de buscar formas de corregir lo que les parece injusto; por ejemplo, aquellas desigualdades que no tienen relación con el mérito, el esfuerzo o el talento. Del mismo modo, reconoce que naturalmente nos compadecemos de la miseria de los menos aventajados de la sociedad. No es sorpresivo, entonces, que activemos ciertos mecanismos redistributivos. Se trata de respuestas similares a las que proporciona Rawls (1971) en su seminal teoría de la justicia: las desigualdades serán aceptables si son el resultado de una competencia abierta a todos los participantes -igualdad de oportunidades formal y efectiva- y si operan en favor de los menos aventajados (el llamado principio de diferencia). Fischer tiene reservas respecto del principio de igualdad de oportunidades, pues, como vimos, piensa que en general los padres tienen el derecho de traspasar a sus hijos ciertas ventajas obtenidas en la vida. Pero parece estar dispuesto a hacer concesiones al respecto. Por su parte, Rawls tampoco ignora el papel que juegan los incentivos — para mejorar estatus, diría Fischer - en la vida social. Sostiene que siempre será preferible una sociedad cuya distribución sea igualitaria salvo que una distribución desigual mejore las condiciones de los más vulnerables. Es decir, prefiere la distribución desigual porque reconoce que la distribución igualitaria anularía el espíritu emprendedor y creador de riqueza que impacta positivamente en el bienestar agregado, y especialmente en los más pobres. Ésa es, precisamente, la crítica que recibe el proyecto rawlsiano desde la izquierda. En cualquier caso, Rawls pensaba que nuestras posiciones sociales no son realmente merecidas. Fischer insiste que están fuertemente vinculadas a la actitud que exhibimos frente al riesgo, cuestión que parece implausible a la luz de su propia teoría: estamos donde estamos porque les permitimos a nuestros antecesores traspasar sus ventajas a las nuevas generaciones. 
Subsiste, sin embargo, una tensión más importante entre la teoría de Fischer y el liberalismo estrictamente político, el segundo gran proyecto de Rawls (1993). Este último indica que el contenido de los esenciales constitucionales y asuntos de justicia fundamental no será determinado por doctrinas particulares y comprehensivas de la vida buena, sino por elementos comunes a las doctrinas — razonables - que estén presentes en una sociedad pluralista, aquello que Rawls denomina "consenso traslapado". Del mismo modo, para participar en la discusión de dichos contenidos, los ciudadanos deben idealmente esgrimir razones públicas; esto es, razones que no sean exclusivas de una doctrina ética o metafísica controvertida. La pregunta crucial, en este sentido, es si acaso los postulados de Fischer no están descansando en una filosofía naturalista (o materialista) tan controvertida como puede serlo una doctrina religiosa. Puesto de otro modo: si la psicología evolutiva representa un conjunto de ideas que pueden ser clasificadas como doctrina comprehensiva, en la medida en que está asociada a una serie de presupuestos cosmológicos, teorías epistemológicas y proposiciones metaéticas, entonces quedaría descalificada para estructurar los principios políticos en una sociedad pluralista. Las razones que ofrecería esta filosofía naturalista, en terminología rawlsiana, no serían públicas. Es decir, la perspectiva darwiniana no sería una epistemología compartida sino un credo combatiente.

La respuesta de Fischer podría reconstruirse de la siguiente forma: hay que distinguir entre ciencia e ideología. Las doctrinas comprehensivas controvertidas a las que se refiere Rawls son básicamente ideologías. Pero la psicología evolutiva es ciencia. Por tanto, no compite de igual a igual con la religión u otras perspectivas filosóficas como el marxismo, el kantismo o el utilitarismo. En teoría, es un argumento legítimo: el propio Rawls (1993) reconoce que el consenso científico forma parte del arsenal de razones públicas que podemos ofrecer a nuestros conciudadanos para fundar la legitimidad política. El peso de la prueba recae entonces en la escuela de Fischer: les toca a ellos demostrar por qué el saber construido a partir de los hallazgos de Darwin y sus herederos forma parte del acervo indisputable de la ciencia (cuestión que no me atrevo a afirmar). Si lo consiguen, entonces la psicología evolucionaria calificaría efectivamente como ciencia y no como ideología. Gozaría entonces de un estatus epistemológico privilegiado en la arquitectura de justificación política según las reglas del propio liberalismo rawlsiano. 


\section{SOCIALISMO COMO MALADAPTACIÓN}

Álvaro Fischer es un seguidor de las teorías del antropólogo francés Pascal Boyer, sobre el origen de la mentalidad religiosa y la persistencia del fenómeno en las sociedades humanas. Boyer (2001) intenta explicar la religión desde una perspectiva científica. Pues Fischer intenta hacer lo mismo con el socialismo. La estrategia es interesante, pues el objeto de estudio no es rechazado en tanto moralmente perverso, como lo hacen muchos ateos respecto de la religión. Fischer tampoco buscar desprestigiar al socialismo, sino explicar científicamente su penetración en el debate político. Es decir, del mismo modo que Boyer parte de la base de que los dogmas de la religión no son ciertos pero su vigencia es científicamente explicable, Fischer parte de la base de que el socialismo está equivocado, pero hay buenas razones —alojadas en nuestra estructura cognitiva - para entender su vitalidad. Fischer describe al socialismo como un meme, pero creo que su idea queda mejor plasmada en el concepto de maladaptación: el socialismo obedecería a un impulso primario de nuestra especie, encriptado en nuestra herencia genética, que no se adaptó correctamente a los cambios en el entorno. Ese impulso se refiere fundamentalmente a la tendencia humana a la cooperación. Es el ámbito en el cual no es tan relevante la retribución por el esfuerzo, pues todos recibirán lo necesario para su subsistencia con independencia de la contribución individual. Es lo que ocurre, advierte Fischer, en los núcleos familiares. Los miembros de una familia se comportan comúnmente de manera altruista, incluso beneficiando a otros miembros a costa propia. La solidaridad entre parientes es la regla y no la excepción. Del mismo modo, era la regla cuando las comunidades de sapiens eran del tamaño de una gran familia y todos se conocían con todos. En ese escenario, la cooperación era enteramente natural y resultaba, por tanto, razonable que las recompensas sociales se distribuyeran en forma más o menos equitativa. La tendencia competitiva, en cambio, estaba reservada para los miembros de la tribu adversaria. En otras palabras, se cooperaba en el estrecho círculo de conocidos y se competía en el anonimato de los desconocidos. El fracaso del socialismo, según esta tesis, se explica en su incapacidad de aceptar que en la actualidad vivimos en sociedades extensas y complejas en las que nadie se conoce y, por ende, no alcanzan a desarrollarse los vínculos afectivos 
que dan origen al altruismo. En otras palabras, el socialismo está muy bien para grupos chicos en los cuales la cooperación es ley social. El capitalismo de mercado, en cambio, sería el adecuado para dar rienda suelta a los instintos competitivos que despiertan en condiciones de impersonalidad.

La tesis puede explicarse aun de otro modo: todos tenemos tendencias cooperadoras y tendencias competitivas. Somos altruistas en ambientes sociales, como la familia, y egoístas en los ambientes impersonales, como los mercados. La idea estaba presente ya en Darwin, quien no pensaba que la supervivencia del más apto fuera pura competencia. La noción del más apto se refería justamente a la aptitud de los organismos individuales para combinar ambos impulsos conductuales: a veces convenía la cooperación, a veces convenía la competencia. El error fatal del socialismo, según Fischer, es creer que las tendencias cooperadoras que la evolución labró para ambientes sociales reducidos puedan imponerse coercitivamente en todos los escenarios. En este sentido, Fischer menciona pertinentemente el trabajo del filósofo socialista Peter Singer. La izquierda, escribió Singer (1999), también debe ser darwiniana: debe tomar en serio la evidencia de que cargamos con una herencia que no se manifiesta sólo en nuestra anatomía y ADN, sino también en nuestro comportamiento. Es decir, que hay ciertos rasgos de nuestra naturaleza que no debieran ser discursivamente descartados como meras invenciones culturales o estrategias de opresión deliberadas. Por el contrario, piensa Singer, la evidencia de las ciencias naturales debiera servir de referencia para construir propuestas políticas, sin abandonar el norte normativo de construir un mundo más equitativo. Por ejemplo, el hecho de que las personas actuarán — querámoslo o no- competitivamente bajo ciertas circunstancias, que la búsqueda de estatus social es una marca indeleble de nuestro negocio reproductivo, que la tendencia a favorecer a nuestros parientes puede ser morigerada y regulada (pero nunca erradicada), que distintas formas de cooperación social son posibles en la medida en que sean percibidas como beneficiosas para todos, etcétera. En resumen, que el socialismo debe aprender a identificar y respetar el trabajo de la evolución.

La tesis que presenta Fischer también podría ser presentada de la siguiente forma: la cooperación está en el corazón del pensamiento político de izquierda y la competencia en el corazón del pensamiento polí- 
tico de derecha. Siguiendo el razonamiento expuesto, todos seríamos de izquierda $y$ de derecha: de izquierda entre familiares y amigos - porque la cooperación es más eficiente en el mundo de las relaciones sociales - y de derecha entre desconocidos — porque la competencia es más eficiente en el mundo de las relaciones productivas-. Como la política, especialmente la económica, organiza la sociedad en su dimensión macro, sus reglas tienen que reconocer la persistencia del ánimo competitivo. Si el socialismo insiste en la exigencia coercitiva de la cooperación - por ejemplo, a través de un sistema previsional de reparto- se va a estrellar contra un muro irreductible y las personas finalmente buscarán la manera de darles salida a sus impulsos. Aquí aparecen en toda majestad las limitantes que la psicología evolutiva impone sobre los debates normativos, pues "para proponer doctrinas políticas, diseñar instituciones que rijan la vida social o formular políticas públicas que utilicen recursos y esfuerzos de todos", piensa Fischer, "es necesario tomar en cuenta el estado del arte más actualizado que tengamos sobre la naturaleza humana" (247).

\section{REFERENCIAS}

Arnhart, L. 2005. Darwinian Conservatism. UK y USA: Imprint Academic.

Boyer, P. 2001. Religion Explained: The Evolutionary Origins of Religious Thought. Nueva York: Basic Books.

Fischer, A. 2017. De naturaleza liberal. Cómo la moral y el comportamiento humano tienen su mejor expresión en una sociedad liberal moderna. Santiago: Catalonia.

Haidt, J. 2012. The Righteous Mind. Why Good People are Divided by Politics and Religion. Londres: Penguin Books.

Hayek, F.A. 1960. The Constitution of Liberty. Chicago: University of Chicago Press.

Hume, D. 1739. A Treatise of Human Nature. Londres: John Noon.

Midgley, M. 2002. Evolution as a Religion. Nueva York: Routledge.

Nozick, R. 1974. Anarchy, State and Utopia. Oxford: Blackwell.

Rawls, J. 1971. A Theory of Justice. Cambridge, MA: Harvard University Press. 1993. Political Liberalism. Nueva York: Columbia University Press.

Ridley, M. 2012. Adam Darwin: Emergent Order in Biology and Economics. Conferencia en el Adam Smith Institute.

Shermer, M. 2006. "Darwin on the Right: Why Christians and Conservatives Should Accept Evolution". Scientific American, octubre 1. 
Singer, P. 1999. A Darwinian Left: Politics, Evolution and Cooperation. New Haven: Yale University Press.

Wilson, E.O. 1998. Consilience: The Unity of Knowledge. Nueva York: Knopf. EP 